\title{
Dispositif de Normes et diagnostic de handicaps
}

\author{
Jacqueline Descarpentries
}

Dans cet article " Le handicap est d'abord pensé comme relatif à une déficience provoquant une incapacité - permanente ou non - puis il est redéfini relativement à une inégalité de moyen, en intégrant l'idée que "des interventions destinées à lever les obstacles environnementaux et sociaux sont nécessaires ${ }^{1 "} "$.

\section{Introduction}

"Si le concept de norme peut être employé dans le domaine de la théorie sociologique où il désigne une contrainte objective n'ayant pas sa source dans l'individu mais dans le fait de l'existence collective " (Legrand, 2007)2, il peut aussi décrire l'opposition entre deux systèmes polarisés à une réalité posée comme contre-valeur à partir d'un normal préalablement conçu. L'anormal sera ainsi, comme le formule Georges Canguilhem

" existentiellement premier "3. Dans ce sens, je mets ici en discussion autant " la puissance " des normes que le seul pouvoir des normes. Même si les deux types d'interprétation sont différents et même opposés, ils ne peuvent être confondus : la dynamique de la puissance est immanente en ce sens qu'elle présuppose une simultanéité de la cause et de l'effet qui sont alors dans un rapport de détermination réciproque ; alors que la référence à un pouvoir implique une transcendance, réalisée par le moyen d'une antériorité de la cause par rapport à l'effet. La question que je souhaite alors aborder ici, ne fût-ce qu'à titre préliminaire, est de savoir comment le diagnostic du handicap construit à partir de la norme biologique, induit une norme doxique de normativité sociale inscrite autant dans le registre des technologies de normation des corps par les agents chargés de dresser ou de redresser des corps dans le registre des éprouvés du corps vécu handicapé. Le sujet est vaste ; aussi, cette contribution se veut ouvrir un débat, elle est provisoire et incomplète. II est nullement 
question de traiter " la question de la norme chez Michel Foucault et chez Georges Canguilhem qui ont tous les deux accordé une extrême importance aux interrelations du naturel et du culturel, du biologique et du social » (Macherey, 2009)4, mais de réfléchir à l'usage du concept de norme travaillé à partir de ces deux approches philosophiques dans l'histoire des idées sur l'anormalité pour appréhender l'usage social et politique du diagnostic de handicap entendu comme un dispositif.

\section{La normalisation des discours sur le handicap du corps de l'autre}

Un discours et les pratiques discursives qui y sont associées, sur le handicap, sont toujours liés à une époque et aux formes associées de savoirs et de pouvoirs de la normalisation des discours et des pouvoirs des institutions qui s'en emparent. En effet, un système de savoir reflète un système de pouvoir (selon Kremer-Marietti en 19745) par une mise en scène des schèmes pré-conceptuels déterminant la régularité selon laquelle se forment les concepts dans une configuration de discours. C'est-à-dire qu'il advient une intelligibilité à la normalisation des discours sur le handicap par la description, la spécification et la localisation d'une régularité de la norme biologique désignée par la puissance de production des normes médicales qui ont un effet de pouvoir sur la prise en charge des personnes en situation de handicap.

En effet, en France, la personne handicapée physique ou psychique est institutionnellement et politiquement étroitement dépendante des politiques publiques de santé. Elle est communément insérée dans des dispositifs médicaux et/ou éducatifs à partir des classifications du handicap dans des établissements sanitaires et sociaux agréés par L'État. Tout un chacun s'accorde, autour d'un discours sur la fonction sociale du diagnostic du handicap, pour déterminer, orienter, le corps handicapé selon des classifications établies à partir du diagnostic de handicap justifié par les résultats des argumentaires scientifiques de la ou des pathologies qui déterminent les normes d'intervention sur les corps de la personne handicapée.

Les mesures épidémiologiques fondent effectivement les connaissances sur la pathologie et classifient les handicaps, relayées par des instances sociales laissant peu d'espace à l'initiative du sujet handicapé. Une logique hétéronomique suggère en effet qu'il existe une statistique exhaustive entre les faits biologiques, physiques et psychiques et sur tous les évènements de vie pour établir toutes les corrélations possibles et affirmer un lien de causalité entre les facteurs de diagnostic et le devenir probable de l'individu handicapé. Tout se passe donc comme si la corrélation était un lien de causalité issu de la connaissance du handicap comme une expérience aléatoire, dont les déterminants d'amélioration du vécu du handicap restent de l'ordre du probable, " du risque à vivre " construite dans la norme des maladies de l'homme normal. La norme pathologique est ainsi présentée comme " donnée préexistante 》 de la normativité biologique qui a le pouvoir de statuer sur la normativité sociale du handicap, mais aussi sur la personne handicapée contrainte de se soumettre, tout au long de sa vie, au pouvoir exercé par le travail médical, sanitaire et social sur son corps handicapé entré dans la catégorisation de norme des maladies de l'homme normal.

Placé dans une injonction paradoxale entre un handicap diagnostiqué et une injonction à la norme qui exclut la personne diagnostiquée handicapée de " la vie normale ", force est alors de constater que les logiques à l'œuvre sont non seulement celles de la 
mathématisation de la vie, mais aussi une mathématisation du risque à devenir normal qui justifient les prises de décisions en matière de protection ou d'exclusion " de la vie normale " des populations handicapées. Même si au demeurant la gestion sociale des populations handicapées est socialement acceptable et acceptée par tous les citoyens, voire demandée par eux, le diagnostic de handicap légitimé par « la norme de la science " (selon un postulat de rationalité scientifique) agit non seulement comme norme de moralisation de la gestion du corps de l'autre vulnérable, mais aussi comme une mise en conformité au normal pathologique. Même si des études récentes démontrent le caractère discriminant et discriminatoire des prises en charge du corps handicapé (Chauvière, 2003)6, tout se passe alors comme cette raison de cette rationalité légitime toute forme d'intervention sur le corps handicapé à laquelle le sujet handicapé doit se soumettre.

\section{La normativité du discours des maladies de l'homme normal}

En effet, les formes discursives de la rationalité, qui désignent la santé, la pathologie, le risque, le handicap, l'anormalité sont toutes issues de travaux de faits scientifiques sur le corps du sujet anatomo-centré, mais aussi du sujet épistémique, objet de pratiques cognitives objectivées qui permettent de justifier les pratiques d'intervention médicale, éducative ou psychothérapeutique sur le corps du sujet diagnostiqué handicapé. Le corps vivant handicapé, désigné à la troisième personne (un corps décrit), est en effet créé par cette indécision ontologique du sujet à la fois objet de la mesure de la santé, de la pathologie, de son dénombrement et de son démembrement à travers nos moyens discursifs de la construction de la norme biologique et anatomique du corps grâce au social pathologique et le savoir sur "le risque à vivre " comme une déviance à la norme biologique. Mais dans la mesure où il n'existe pas, en-dehors de leur construction sociale, de fondement naturel à la norme, pas plus qu'il n'existe de fondement culturel des vies, cette indécision ontologique pour le sujet handicapé le contraint de fait à incorporer les normes du normal par une prise en charge thérapeutique ou éducative empreinte d'une crise anthropologique des vies humaines dans la société contemporaine française. En effet, les populations handicapées sont soumises à une injonction de mise en conformité à la normalité sociale qui passe par l'autonomie, qui précise la relation entre la vie sociale normale et l'individualité (Leblanc, 2000)7. Elles sont effectivement appelées à vivre autant dans un contexte marqué par les normes sociales des maladies de l'homme normal que dans les micro-normes à incorporer d'une vie ordinaire normale visant la responsabilité et l'autonomie ; alors que le diagnostic de handicap les inscrit de fait dans l'exclusion des normes étalons de l'homme normal (Leblanc, 2007)8 ! Elles se doivent autant d'être porteuses d'opportunités d'autonomie visant la construction d'un soi responsable dans le jeu discordant des maladies de l'homme normal (Ibid.), que d'incorporer la disqualification sociale du soi handicapé, devenu, si ce n'est un sujet de la vulnérabilité, mais surtout un objet de la discrimination puisque anormales ! Le diagnostic de handicapé, par le jeu de nos relations aux disqualifications sociales négatives, entre dans un hors norme qui est conféré à leur existence (Butler, 2005)9, reniant toute une multiplicité de différences dans les pratiques corporelles du corps handicapé ; alors que comme le rappelle Georges Canguilhem10, la maladie reste un trouble qui naît de la permanence de l'état 
normal... En effet, si la norme et la valeur ont un sens équivalent pour Canguilhem, ce dernier nous oblige à repenser nos interprétations normatives des catégories fondamentales du handicap car elles définissent bien moins la maladie, mais bien plus la normativité intrinsèque de la vie ; autrement dit, la puissance de la vie dans la norme pour nommer la vie et le handicap. Le physiologique du pathologique étant une valeur biologique, une norme immanente de la vie, comme le rappelle Élodie Giroux (2010)11, il ne peut donc y avoir de pathologie objective dans le sens où, son objet est toujours un import d'origine subjective et n'est pas tant un fait qu'une valeur -si, bien évidemment, la dimension existentielle du diagnostic de handicap ne peut être niée- de par la prétention à l'objectivation ignorant l'essentielle normativité du vivant sain ou malade au profit des capacités du sujet.

En effet, le pathologique étant une normativité restreinte, une réduction de l'assurance de la biologique initiale, la personne diagnostiquée handicapée peut aussi être en

" bonne santé " en référence à Georges Canguilhem, car la santé est d'abord le contraire vital du pathologique qui désigne la capacité des êtres vivants à surmonter la maladie, le stress et les modifications du milieu de vie en créant leurs propres normes ; le handicap, la maladie, la santé est donc " une vérité du corps " (Canguilhem, 1990)12 entre expérience et connaissance de la santé et la maladie. Ainsi, d'un point de vue anthropologique, le diagnostic du handicap, dont le rôle social est de participer à la séparation entre les sujets normaux et les sujets anormaux (Foucault, 2003)13, est issu des connaissances et des savoirs de l'épidémiologie de laboratoire, qui n'est autre que le produit des technologies variées de la mathématisation de la vie et des épistémologies de la recherche sur le risque à vivre normalement, justifiant socialement et politiquement, voire économiquement, la normation des comportements des populations handicapés à partir, non seulement de la connaissance du corps normal et anatomo-normé et de la norme biologique du débile corporel ou psychique ; mais aussi de la norme éducative qui agit comme une technologie de pouvoir de normation sur le corps handicapé à partir d'un guide de bonnes pratiques qui peut devenir le panoptique de surveillance des corps handicapés.

\section{La normalisation des discours et normation du corps handicapé}

Dans l'épistémologie de Michel Foucault, la communauté scientifique s'accorde à la succession de deux modèles d'analyse de la norme. Le premier s'applique à comprendre les modalités selon lesquelles s'effectuent la normalisation du discours, le second se portant vers une généalogie critique de la normation des conduites dans leur élément concret matériel et pratique. II s'agit en fait de l'ensemble

" des codes et règles de dressage et de contrôle qui détermine qui est inapte et qui ne l'est pas, qui est normal et qui est anormal. L'opération de la normalisation disciplinaire consiste donc à essayer de rendre les gens et les actes conformes au modèle, le normal étant précisément ce qui peut être conforme à la norme. La norme a donc un pouvoir descriptif. C'est pourquoi pour Foucault les techniques disciplinaires font plutôt référence à une normation plutôt qu'une normalisation » (Nicaine, 2014) ${ }^{14}$.

Ainsi, dans l'institutionnalisation du diagnostic du handicap, on peut légitimement se demander comment se construit l'usage social des savoirs sur le handicap qui agit comme une technologie disciplinaire de normation qui a un effet de pouvoir sur les 
pratiques discursives et prend sens autant à travers la normativité intra-discursive déterminée par des dépendances dans les relations d'implication des savoirs déterminées par l'histoire, qu'à travers des systèmes de dépendance extra-discursive entre les transformations des savoirs et les changements économiques, politiques et sociaux. L'usage social des savoirs de diagnostic de handicap transforment en effet les conditions objectives de l'existence des personnes handicapées en modifiant la manière dont les discours existent et peuvent exister dans leur réalité matérielle. Ainsi, les dispositifs de diagnostic de handicap créent un ensemble de complexes de la normalisation de la vie anormale à travers tout un ensemble de processus infra-légaux de la régulation des conduites comme toutes les structures sociales et contemporaines d'accueil des personnes diagnostiquées, lieu signifiant des normes à incorporer par l'orthopédie éducative. On peut notamment nommer les formations de professionnels chargés de les éduquer. La question est effectivement de savoir comment les professionnels du secteur sanitaire et social réutilisent les contenus des savoirs construits par le biopouvoir qui déterminent le diagnostic du handicap pour faire œuvre spécifique de dressement ou de redressement du corps handicapé en se saisissant des dispositifs éducatifs issus de la pensée critique, c'est-à-dire des dispositifs éducatifs centrés sur les outils de développement du néolibéralisme et occidentalo- centré. $\mathrm{Ce}$ n'est pas le moindre des paradoxes si l'on admet que le travail d'accompagnement du corps handicapé vise non seulement la réduction des risques de détérioration de l'état de santé initial par leur prévention, mais aussi l'amélioration du risque à vivre par une représentation plus ou moins vague de la " bonne norme de santé " selon laquelle le sujet handicapé est sensé adhérer pour être autonome et responsable, selon la bonne vieille logique rawlsienne de la justice sociale !

En effet, force est de constater qu'un ensemble de procédures, de techniques des normes du biologique et de normes éducatives renvoie à tout un ensemble de pratiques éducatives ou d'intervention éducative sur le corps du débile corporel ou psychique vers des processus d'incorporation de normes sociales construites à partir des cadres normatifs de la classification du handicap, mais aussi de la philosophie nord- américaine de l'éducation. Ces deux processus se combinent. D'une part, les éléments d'expertise et des faits scientifiques du diagnostic de handicap sont socialement et politiquement utilisés pour justifier toutes les formes de dressement ou le redressement du débile corporel ou psychique, qui va de la classification des établissements (ITEP... MECS... etc.) aux formes de prise en charge ; d'autre part, au nom des principes de la justice sociale, tout un ensemble de valeurs associées à l'éducation comme à l'autonomie et à la responsabilité, se trouvent au cœur des pratiques éducatives de professionnels développées dans des schémas d'analyse incapables de rendre compte de leur efficacité dans l'amélioration des conditions de vie des personnes en situation de handicap, puisqu'ils sont inscrits dans des cadres d'analyse politique, analytique et conceptuel du présent, créés sur la base de processus politiques néolibéraux nécessaires à leur développement.

En partant de là, on peut légitimement se demander quelles sont les marges de manœuvre des professionnels sanitaires et sociaux placés entre l'oppression politique exercée par les formes du biopouvoir et de la biopolitique par les normes de diagnostic du handicap comme une normation par le social sanitaire du corps handicapé ; et les dispositifs éducatifs devenus des technologies de normalisation et de domination néolibérales nécessaires à son développement.

L'action sanitaire et sociale ne ferait-elle pas ainsi un usage social du handicap pour 
atteindre des buts politiques et culturels quelle que soit la manière dont on les définit. Le diagnostic de handicap n'est donc pas " une simple » reproduction de performativité du pouvoir épistémique de l'objectivité du diagnostic médical, mais il est aussi marqué par les limites d'un espace social constructiviste de la norme biologique et par là même de la vulnérabilité des corps comme un processus de construction sociale et biologique de la norme, dont le mode de fonctionnement est à la fois interactif et résistant aux postures épistémologiques structuralistes, participatifs et pourtant distincts de leur paradigmes, revendiquant à la fois la subjectivité corporelle et l'égalité de " traitement social des vulnérabilités " et la différence d'une représentation politique sociale de la diversité des handicaps tout en insistant sur la spécificité de la norme.

\section{En conclusion : un surcode structuré}

Une conclusion ouverte s'impose autour du diagnostic du handicap comme un surcode structuré par le biopouvoir qui met en évidence les modes de rationalités de la norme médicale, éducative et sociale. Ce " surcode structural » est dilué dans un ensemble complexe du système social du diagnostic à la prise en charge médicale, éducative ou psychothérapeutique dans un nombre important de sous-systèmes en connexion. II participe de fait au maintien d'un système de code non seulement linguistique propre au travail social, mais aussi comme code social puisque du diagnostic, des glissements de rationalités vont s'opérer entre le scientifique, le social et le politique pour construire l'accompagnement ciblé pour ces populations dont la finalité est de maintenir l'ordre social au nom des principes éthiques et moraux entre les normaux et les anormaux. Le pouvoir effectif de ce surcode structural du diagnostic du handicap est de faire émerger différentes strates de décisions issues des glissements de rationalités entre le biologique et le social, inscrivant le corps de la personne handicapée dans une soumission à l'ordre établi entre le corps normal et le corps anormal avec pour projet éducatif un redressement des corps pour vivre une vie normale ! Ainsi, les critères de diagnostic du handicap désignent non pas ce processus, mais une position enclavée de groupes de populations anormales inscrites dans un rapport social de sujets dominés par l'anormalité définie par les instances politiques du biopouvoir.

En effet, si la notion d'histoire effective de Michel Foucault ne cherche pas à recouvrer les évènements et les acteurs des procédés des opérations "d'implantation ", il n'en demeure pas moins que les opérations de pouvoir à l'œuvre au sein du discours pathologisant sur le handicap produit la formation d'un sujet anormal porteur d'une subjectivité déviante à la norme de l'anormalité. Le diagnostic de handicap considère en effet le corps vivant handicapé comme la déviance "normale " qui assure alors le bon fonctionnement du système sanitaire et social et son auto-reproduction. Si le handicap est ainsi issu de ce discours des normes d'un socius produit, légitimé et prescrit par une fausse déviance issue d'un surcode structural qui travaille à entretenir les connexions entre les éléments du système pour maintenir le système, on comprend bien la puissance de la norme dans le diagnostic du handicap illustré par le pouvoir de la normation de la bio corporéité effectivement confrontée à l'archéologie de l'ambiguïté des liens entre le gouvernement des corps 15, véhiculant le conditionnement normatif du corps humain et sa protection en qualité de sujet vulnérable hors norme. II est particulièrement questionnant que les points de rupture entre des idéologies antagonistes comme se situant au niveau de la relation corps/nature, corps/culture, 
voire corps/technologie, ne supportent pas depuis longtemps l'interrogation d'ordre foucaldien : " Comment le diagnostic du handicap s'est-il constitué en tant qu'interprétation de normes régulatrices d'un modèle naturel du corps humain en légitimant son intervention de normalisation sur les corps ? Si, dans ce sens, Michel Foucault consacre un cours, en 1974-1975, aux « anormaux " 16 et traite précisément de la genèse de cette problématisation, nous nous emparerons des chapitres 6 à 8 où il isole trois figures des " anormaux » et pose la question de leur place dans la société du Xlle et XIXe siècle : le monstre, l'individu à corriger et l'onaniste.

Quarante ans plus tard, nous pouvons nous demander si ces figures survivent encore et si elles sont toujours un avatar de la technique de l'aveu contraint et obligatoire et de la confession telle qu'établie par la religion dans ses aspects propres à la question de la chair, resté en quelque sorte l'arrière-fond de toutes les techniques et examens médicaux pour définir l'anormalité et le handicap. Comment Michel Foucault aurait-il analysé "les monstres à enfermer", entre ce qui relève du handicap physique, psychique, social, ceux qui présentent une dangerosité criminologique et du risque du passage à l'acte, les « individus à corriger » dans le développement des neurosciences et de la crise sans précédent de la psychiatrie française qui, plus que jamais, est le lieu du contrôle des agirs des individus, catégorise, classifie, inclut/exclut ceux qui sont aptes à participer à l'ordre social et ceux qui ne le sont pas dans une politique ultralibérale du healtism liée au plein développement de l'industrie pharmaceutique ? En quoi la figure de l'onaniste du XVIIIe éclaire notre présent quant elle était une certaine technique de conscience orientée vers la lutte contre la masturbation et ses effets prétendument pathologiques, l'onaniste n'est-il pas la personne handicapée dont la sexualité est à dresser ou à redresser par une éducation à la sexualité hétéro-normée et postcoloniale ?

\section{BIBLIOGRAPHIE}

Andrieu, B. (2008). Devenir hybride. Nancy : PUN.

Agamben, G. (2014). Qu'est-ce qu'un dispositif ? Paris : Rivages, coll. « Rivages poche ».

Butler, J. (2005). HumaIN et inhumaIN, LA critique des normes, Entretiens. Paris : éd.

Amsterdam. Bury, J.-A. (1988). L'éducATION pour IA SANTE, enjeux, concepts et méthodes.

Bruxelles: De Bœck. Cadore, B. (1994). L'expérience bioéthique de IA responsABILité.

Louvain-la-Neuve : Artel.

Descarpentries, J. (2000). Essai de théorisation des modèles éducatifs dans le champ de la santé.

Spirale, (25), Éducation pour la santé s/d Jacqueline Billon, Université de Lille III.

Fassin, D. (1992). Anthropologie et santé publique. CaHIEr des sciences

huMAINES, 28. Fassin, D., \& Memmi, D. (2004). Le gouvernement des corps.

Paris : EHESS.

Foucault, M. (1966). Les mots et les choses. Une ARchéologie des sciences huMAINEs. Paris :

Gallimard. Foucault, M. (1969). L'ARchéologie du SAVOIR. Paris : Gallimard. 
Foucault, M. (2003). Les anormaux, Cours aU collège de France. Paris :

Gallimard. Foucault, M. (2008). Le gouvernement de soi. Paris : Gallimard.

Genard, J.-L. (1999). La grammalRe de IA responsabllité. Paris : éd. du Cerf.

Heilbrum, B et AL. (2004). La performance, une nouvelle idéologie. Paris : La Découverte.

Janssen, T. (2006). LA solution intérieure, Vers une nouvelle médecine, du corps et de l'esprit. Paris : Fayard.

Jonas, H. (1990). Le principe responsabILité, une éthique pour IA civilISATION technologique. Paris, Cerf. Lecourt, D. (1996). Le paradoxe moderne de l'éthique. Le MAGAZINE littérAlRe, 112- 115.

Leblanc, G. (2007). Les MALADies de l'homme norMAL. Paris : Vrin.

Lebrun, J.-. (1997). Un monde SANS limite, un eSSAI pour une clinique psychaNALytique du SOCIAL. Ramonville: Érès.

Lupton, D. (1996). The imperatif of health: public health and the régular body. New york: Oxford University Press.

Macherey, P. (2009). De Canguilhem À Foucault, IA force des normes. Paris : La Fabrique.

\section{NOTES}

1. . Agamben, G. (2014). Qu'est-ce qu'un dispositif ? Paris : Rivages, coll. «Rivages poche ».

2. . Legrand, S. (2007). Les normes chez Foucault. Paris : PUF, coll. « Théories pratiques ».

3. . Canguilhem, G. (1996). Le normaL et le pATHOLogique. Paris : PUF.

4. . Macherey, P. (2009). De CANGuilhem a Foucault, IA force des normes. Paris : La Fabrique.

5. . Kremer-Marietti, A. (1974). Michel Foucault, Archéologie et généALogie. Paris : Seghers, coll.

"Philosophie », réed. Paris : Le Livre de poche, coll. " Biblio/Essais », 1985.

6. 6. Chauvière, M. (2003). Handicap et discriminations. Genèse et ambiguités d'une inflexion de l'action publique. In D. Borrillo (Dir.), Lutter contre les discriminations. Paris : La Découverte, coll.

«Recherches ».

7. . Leblanc, G. (2000). Lectures de CANGuilhem. Paris : ENS éd.

8. . Leblanc, G. (2007). Les MALADies de l'homme norMAL. Paris : Vrin.

9. . Butler, J. (2005). HumaIN et inhumaIN, LA Critique des normes, Entretiens. Paris : éd. Amsterdam.

10. . Canguilhem, G. (1966). Le normaL et le pATHOLogique. Paris : PUF.

11. Giroux, E. (2010). Après CANGUilhem, définir IA SANTE et IA MALADie. Paris : PUF.

12. Giroux, E. (2010). Op. cit., p. 36.

13. . Foucault, M. (2003). Les ANORMAUX, Cours aU collège de FranCe. Paris : Gallimard.

14. . Nicaise, G. (2014). Foucault : le concept de normation et de normalisation. In LABORATOIRe de IA gouverNANCe, [on line] sur: http://www.lagouvernance.fr/foucault-le-concept-denormation-et- normalisation/.

15. . Fassin, D., \& Memmi, D. (2004). Le gouvernement des corps. Paris : EHESS/Fassin.

16. . Foucault, M. (2003). Les ANORMAUX, Cours AU collège de FranCe. Paris : Gallimard. 


\section{RÉSUMÉS}

Dans cet article, le diagnostic de handicap est analysé comme un dispositif au sens foucaldien, c'est-à-dire " tout ce qui a, d'une manière ou une autre, la capacité de capturer, d'orienter, de déterminer, d'intercepter, de modeler, de contrôler et d'assurer les gestes, les conduites, les opinions et les discours des êtres vivants " (Agamben, 2014) ${ }^{1}$. Autrement dit, je mets en discussion un certain usage social et politique de la norme qui détermine le handicap à travers la rationalité de la normativité biologique et de la normativité sociale des techniques de normation du corps handicapé, du diagnostic de handicap aux formes de prises en charge. J'émets ainsi une hypothèse liée à la mise en place d'une normalisation des discours et des pratiques de prise en charge éducative liée non seulement au diagnostic, mais aussi aux dispositifs éducatifs occidentaux centrés, néolibéraux qui place le sujet du diagnostic dans une injonction paradoxale : placé dans une norme de handicap diagnostiqué, le sujet est censé « devenir normal » par le jeu de l'injonction à l'autonomie et de la responsabilité, malgré la norme de handicap qui exclut l'handicapé de la vie normale dans le dispositif éducatif de normation. Force estalors de constater que les logiques à l'œuvre sont non seulement celles de la mathématisation de la vie et de la mathématisation du risque à devenir normal, induites par les modèles néolibéraux de la marchandisation des corps qui justifient les prises de décisions en matière de protection ou d'exclusion de la vie normale des populations handicapées qui déterminent ainsi les pratiques d'intervention sur les débiles corporels et psychiques.

In this article, the diagnosis of disability is analyzed as a device in the Foucauldian sense, that is to say, "all that has, in one way or another, the ability to capture, direct, determine, to intercept, to model, to control and to ensure the gestures, the conduct, the opinions and the discourses of the living beings "(Agamben, 2014). In other words, I am discussing a certain social and political use of the norm that determines disability through the rationality of biological normativity and social normativity of normative techniques of the disabled body, from the diagnosis of disability to the forms of catch in charge. I thus put forward a hypothesis linked to the establishment of a standardization of discourse and practices of educational management linked not only to diagnosis, but also to the educational devices Western-centered, neoliberal which places the subject of the diagnosis in a The paradoxical injunction: placed in a standard of diagnosed handicap, the diagnosed subject is supposed to "become normal" by the play of injunction to autonomy and responsibility, despite the norm of handicap that excludes the disabled from the normal life In the educational system of normation. It must be noted that the logics at work are not only those of the mathematization of life and the mathematization of the risk to become normal, induced by the neo-liberal models of the commodification of bodies that justify decisionmaking in matters Protection or exclusion from the normal life of the handicapped populations, which thus determine the practices of intervention on the bodily and psychical weaknesses.

\section{INDEX}

Mots-clés : norme, normalité, normation, normalisation, normativité, dispositifs, corps normal, et corps handicapé.

Keywords: norm, normality, normation, normalization, normativity, devices, normal body, and disabled body 


\section{AUTEUR}

JACQUELINE DESCARPENTRIES

Rattachée au Laboratoire Experice, Université Paris 8

Associée au Laboratoire TEC, Université Paris Descartes Sorbonne Cité 